Original Research Paper

\title{
Mobility and Handover Technique in Heterogeneous Wireless Networks
}

\author{
Radhwan Abdullah, Zuriati Zukarnain, Farzaneh Masoumiyan and Azizol Abdullah \\ Department of Communication Technology and Network, University Putra Malaysia, Serdang, Selangor, Malaysia
}

Article history

Received: 18-09-2014

Revised: 16-03-2015

Accepted: 17-03-2015

Corresponding Author:

Radhwan Abdullah

Department of Communication

Technology and Network,

University Putra Malaysia,

Serdang, Selangor, Malaysia

Email: radwanmas@yahoo.com

\begin{abstract}
The management techniques for Mobile IPv6 between different wireless technologies are very important to complete the handover process with the least possible delay. In the fast handover, when a mobile node moves to another network, it needs to do handover operations. These operations have a severe impact on the handover latency. This paper proposes an Enhanced Advanced Duplicate Address Detection (EA-DAD) method in a heterogeneous mobile environment with the support of the MIH services. The proposed method quickly provides a unique Ipv6 address for MNs. At the same time, the binding updates to home agent and correspondent node are to be performed from old access router. We can see through results that by optimizing network layer, EA-DAD quickly presents unique Ipv6 addresses for MNs with a minimum handover latency and packet loss even at high speed movements.
\end{abstract}

Keywords: Vertical Handover, FMIPv6, MIH, Duplicate Address Detection

\section{Introduction}

In IP mobility, there are some important issues related to management techniques between different wireless technologies, these techniques depend on handover operation speed. Mobile IPv6 (MIPv6) is one of the mobility management solutions that can support mobility between different access routers belonging to the same technology (Johnson et al., 2004; Izuan and Zuriati, 2009). This is realized primarily through using Care of Address (CoA) to indicate the location of the Mobile Node (MN). Although the MIPv6 protocol has several promising features and presents an elegant method to support mobility, it has an inherent weakness. That is, during the handover, the MN remains for a short time is unable to connection because of link switching delay and IP protocol operations. Handover is the operation by which an MN maintains an active connection as much as possible when it leaves from old to new networks. The handover operation occurs when the MN changes its network point of attachment from an old access router to a new access router and it should achieve three operations: Movement detection, new CoA configuration and binding update. The $\mathrm{MN}$ is unable to connection as usual during the handover time. The length of this time which is called handover latency is very critical for real-time services. Furthermore, there are many applications which are sensitive to the amount of time in which the $\mathrm{MN}$ remains disconnected while performing a handover operation. This disconnection and delay affects the time of packet delivery within limited period (e.g., VoIP and audio/video streaming applications).

The extended amount of time it takes to complete the handover in MIPv6 has led to the emergence of another protocol which is the Fast MIPv6 (FMIPv6) (Koodli, 2005). In this study, we focus on the design of FMIPv6 protocol. This protocol has been proposed by the Internet Engineering Task Force (IETF). It comes to address the following problem: How to allow an $\mathrm{MN}$ to send packets as soon as it detects a new subnet link and how to deliver packets to an MN as soon as its attachment is detected by the New Access Router (NAR) (Dimopoulou et al., 2005). It reduces the movement detection latency by anticipating handover and preparing the $\mathrm{MN}$ with the information about the NAR before disconnection from the current access router. This information is used to generating and configuration a new CoA by $\mathrm{MN}$ itself that is used to connect with a NAR. The typical address configuration requires Duplicate Address Detection (DAD) (Thomson et al., 2007) to check validity of the configured address. To reduce the binding update latency, a bidirectional tunnel between Old Access Router (OAR) and the NAR is established.

However, it is noted that the DAD procedure easily takes up to $1 \mathrm{sec}$. especially if the DAD begins after the link is created to the NAR. If duplicate CoA occurs, the handover latency will increase greatly. At the same time, we note that the binding updates to the Home Agent 
(HA) and Correspondent Node $(\mathrm{CN})$ are performed after the time point when the MN is IP-capable on the new subnet link (Koodli, 2005). Because of this, the MN communicates with the CN directly via the NAR without using tunnel in a very late time. Thus the packet delay for some packets sent during the handover will be enlarged.

The IEEE 802.21, Media Independent Handover (MIH) (IEEE, 2008) provides generic link-layer intelligence and other network related information to upper layers to optimize handover between different heterogeneous access networks like WiFi, WiMAX and UMTS.

This paper proposed an Enhanced Advanced Duplicate Address Detection (EA-DAD) method with the support of the MIH services. To reduce the latency in the DAD procedure, we let the NAR generates and perform DAD for new CoAs and exchange a number of this address with the neighbour ARs and store this new CoA to the table before to any handover operation. Then when the MN requests the new CoA through the OAR, this new CoA will be distributed to the MN from the OAR. At the same time, to reduce the registration latency in the binding update, the binding update to the $\mathrm{HA} / \mathrm{CN}$ will be performed by OAR. Only a small handover latency, buffering and packet loss are expected when applying the EA-DAD, even with high speed movement.

The rest of this paper is organized as follows: Section 2 discusses some related work. Section 3 describes the main idea of the proposal, EA-DAD with the support of MIH services. Section 4 presents simulation and results while section 5 presents the conclusions.

\section{Related Work}

\section{Fast Mobile IPv6}

When the handover operation happens by changing between different networks, the MN should perform layer 2 handover and Layer 3 Handover. Layer 2 handover is the process with which the $\mathrm{MN}$ changes from one access point to another. Layer 3 handover is the process that the $\mathrm{MN}$ changes the attachment from one access router to another. The layer 3 handover includes three operations: Movement detection, new CoA configuration and binding update. Therefore the handover latency includes three parts:

- L2 handover latency, which is defined as the time interval from the moment that layer 2 link down trigger from the OAR happens to the moment that the layer 2 link up trigger to the NAR happens

- Rendezvous time delay (Vivaldi et al., 2003), which includes two kinds of latency. One is movement detection latency, which is the time interval taken by the MN to detect the presence of a NAR at the new access network and the other is the new CoA configuration latency, which is the time interval taken to configure a new CoA for the $\mathrm{MN}$

- Registration delay, which is the time that it takes to send $\mathrm{BU}$ to the $\mathrm{HA} / \mathrm{CN}$ and the subsequent resumption of communications indicated by a new data packet arriving at the MN from the NAR without passing through the tunnel between NAR and OAR

To reduce the handover latency occurred in MIPv6, FMIPv6 are proposed in (Koodli, 2005) that allows an $\mathrm{AR}$ to offer services to an $\mathrm{MN}$ in order to anticipate an IP layer handoff. The standard specifies two modes of operation namely Predictive and Reactive modes. The major difference between these two modes is on the time to establish the tunnel between the OAR and NAR. The Predictive mode of FMIPv6 is depicted in Fig. 1. By the predictive mode, the tunnel is established before L2 handover, but by the reactive mode, the tunnel is established directly after L2 handover. We focus on the predictive fast handover method in this study because it has shorter latency than the reactive one.

Although FMIPv6 was designed to overcome the major drawbacks of MIPv6 handoff, we notes that if the DAD is not performed at the beginning and is performed after the node has set up a link to the NAR, much time will be wasted if duplicate address exists and such latency cannot be tolerated for latency sensitive applications. On the other hand, we notice that the Binding Update (BU) cannot be started as soon as possible. By the predicative mode, the BUs to $\mathrm{HA} / \mathrm{CN}$ are performed after the link is created to the NAR. After investigation, we find that when the handover starts and the new CoA is known by OAR, the handover procedure will definitely be performed even if there are duplicated addressed or some other situations. Therefore, using this discovery, we propose performing the BU to $\mathrm{HA} / \mathrm{CN}$ beforehand.

\section{Media Independent Handover Services}

Media Independent Handover (MIH) is an emerging IEEE standard which supports seamless handover between homogenous and heterogeneous networks. 802.21 does not in itself implement network handover, rather it provides information to allow handover to and from a range of networks including cellular, GSM, GPRS, WiFi, Bluetooth. The network handover enabling function within the protocol is implemented through the MIH function. The MIH function consists of three elements, the Media Independent Event Services (MIES), the Media Independent Command Services (MICS) and the Media Independent Information Services (MIIS). 


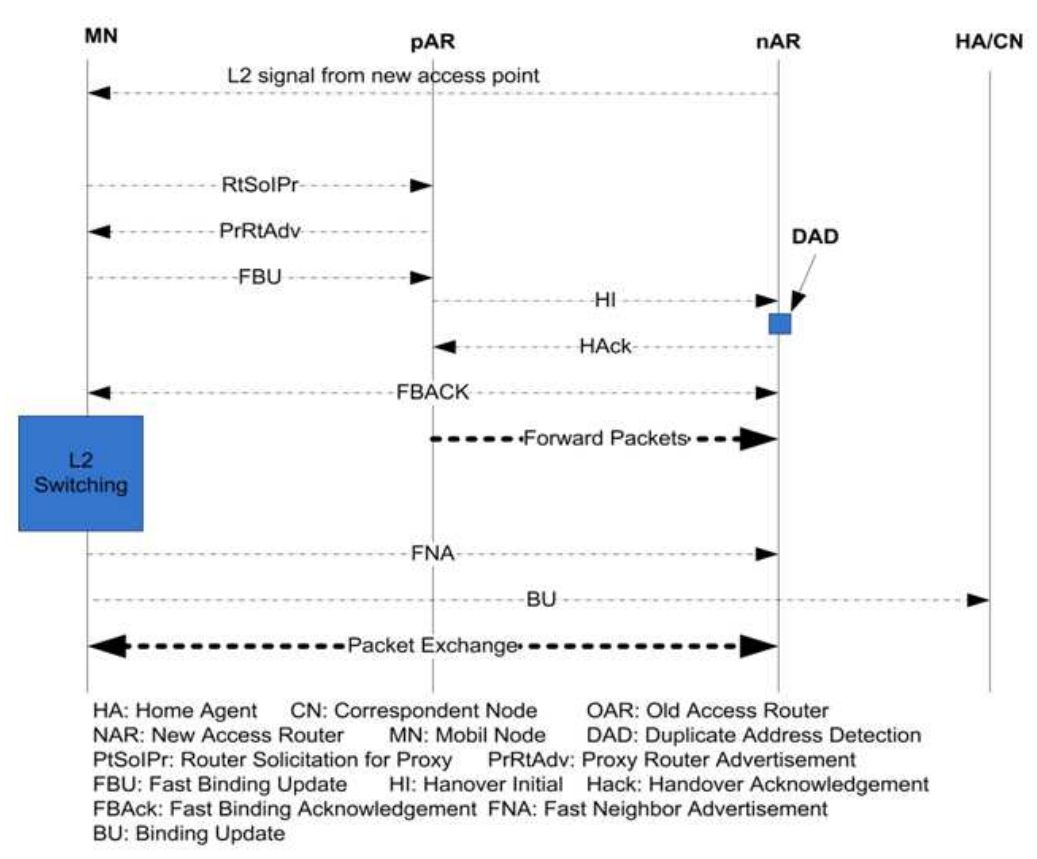

Fig. 1. Handover procedure in predictive FMIPv6

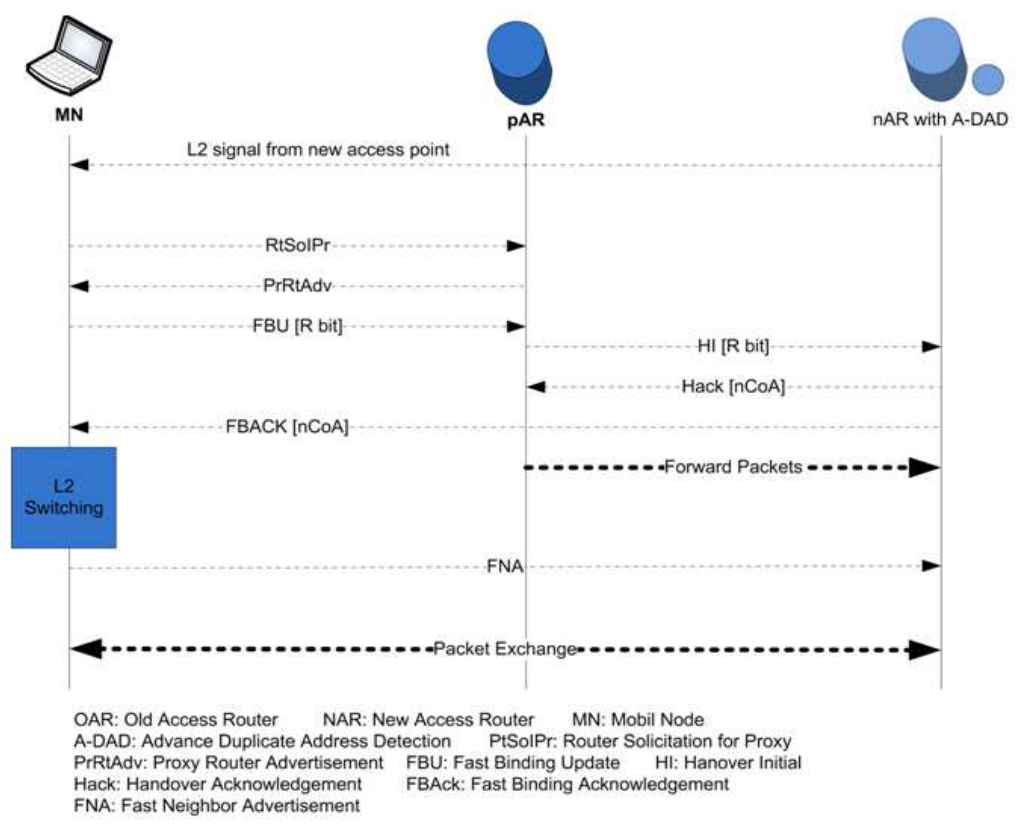

Fig. 2. A-DAD process with FMIPv6

Media Independent Event Services (MIES) will typically be used to detect need for handovers. MIES provides services to the upper layer by reporting event classification, event filtering and event reporting corresponding to dynamic changes in link characteristics, link status and link quality. It reports both local (from the local stack of the mobile node) and remote events (from another MIHF in the network). Some of the common events that have been specified by the standard are "Link Up", "Link Down", "Link Parameters Report", "Link Going Down" and "Link Detected".

Media Independent Command Services (MICS) enables higher layers, locally or remotely, to control the physical, data link and logical link layers. The higher layers control the reconfiguration or selection of an appropriate link through a set of handover commands. 
The commands carry the upper layer decisions to the lower layers. For example the command service may be used to request a mobile node to switch between links. Commands defined include MIH_Switch, MIH_Get_Status, etc.

Media Independent Information Service (MIIS) provides a framework and corresponding mechanisms by which a Media Independent Handover Function (MIHF) entity may discover and obtain network information existing within a geographical area to facilitate the handovers. MIIS primarily provides a set of Information Elements (IEs), the information structure and its representation and a query/response type of mechanism for information transfer. This contrasts with the asynchronous push model of information transfer for the event service. The information may be present in some information server from where the MIH Function in the station may access it.

\section{Improved $D A D$}

Many studies have been presented to eliminate or decrease the time required for DAD. The Advanced Duplicate Address Detection (A-DAD) (Han and Hwang, 2006) technique is one of these studies and it improves on the DAD delay by storing a pool of unique IPv6 addresses at an AR. Each AR generates random addresses as a background process and performs a standard DAD on them. The addresses that are not duplicated are stored in the AR. The AR acts as a passive proxy for addresses. It listens to the neighbour discovery messages from other nodes in the network. If it hears another node performing $\mathrm{DAD}$ on the same address in its pool, the AR silently removes that address from its list and tests a new address to keep the list size constant. A-DAD is used with FMIPv6 as shown in Figure 2. It modifies FBU, HI, HAck and FBAck messages with new options. The MN sends FBU with a new CoA-Request bit (R bit) to the OAR. When the NAR received HI messages containing the same $\mathrm{R}$ bit from the OAR, it immediately takes a unique IP address from its address pool and sends a Hack message to the OAR. As soon as the MN receives such an FBAck from the OAR, it configures and confirms the new CoA. The time it takes for this process will be very short compared with the time for the standard DAD process.

Several other techniques are used to reduce the delay time to get a unique IP address. Optimistic DAD (Moore, 2006) works on the assumption that the probability of a duplicate address is very small. It allows the $\mathrm{MN}$ to use a Tentative Address in some communication while the uniqueness test is conducted by a normal DAD process. Proactive DAD (Tseng et al., 2006) performs DAD on a new IP address before the MN moves to a new network. MLD-DAD (Daley and Nelson, 2003) takes advantage of IIPv6 multicast listener discovery. It assumes all nodes must join a solicited-node multicast address associated with each Tentative Address. So to check the uniqueness of an address, they simply verify whether a node is the first to enter the solicited-node multicast group when it is empty. This method could end up with false duplicates because multiple uncast addresses could join the same solicited node multicast group.

\section{Proposed Method}

Our Enhanced Advanced Duplicate Address Detection (EA-DAD) method will be based on the idea of stateful address configuration. We propose an improved A-DAD method to achieve a fast prediction of the prospective serving network. At the same time, we proposed enhanced FMIPv6 method by binding updates to $\mathrm{HA} / \mathrm{CN}$ are brought beforehand.

\section{Method Descriptions}

EA-DAD similar to A-DAD but in a more efficient manner, EA-DAD separates the unique pool and redistributes it between neighbouring ARs. In more detail, the following steps should be performed beforehand.

- To reduce the DAD latency in FMIPv6 method, each AR randomly generates addresses as a background process

- On generating a new address, each AR must perform its own DAD process to check the uniqueness of the address according to the standard (Thomson et al., 2007)

- If the new addresses are unique, then the ARs that have overlaps in their coverage area will exchange groups of unique addresses and reserve them in the individual address table. These addresses are also considered as unique new CoAs

When assigning an address to a $\mathrm{MN}$, each $\mathrm{AR}$ that has this address must remove it from its table. The AR will generate a new address to keep the number of addresses in the table at a pre-defined value. Here new CoA generation and the DAD performed by AR beforehand to reduce the DAD latency. It is also important to note that except for the decrease on latency, bringing the $\mathrm{DAD}$ procedure forward can make the issued new CoA unique. Thus, the binding update to $\mathrm{HA} / \mathrm{CN}$ can be brought forward because there is no problem on the new CoA.

Our method requires functionalities from the MIIS in form of discovering and selecting the prospective network prior to actual operation of vertical handover without requiring the whole 802.21 to be implemented. Also selecting the prospective network will help the OAR to exchange groups of the unique addresses with prospective ARs. Through this advanced discovery method, a new entry for each discovered AR is created in the OAR and the table will be updated accordingly. Based on the selected information and the copy of the unique address of each network, the ARs are classified and sorted in the table. 


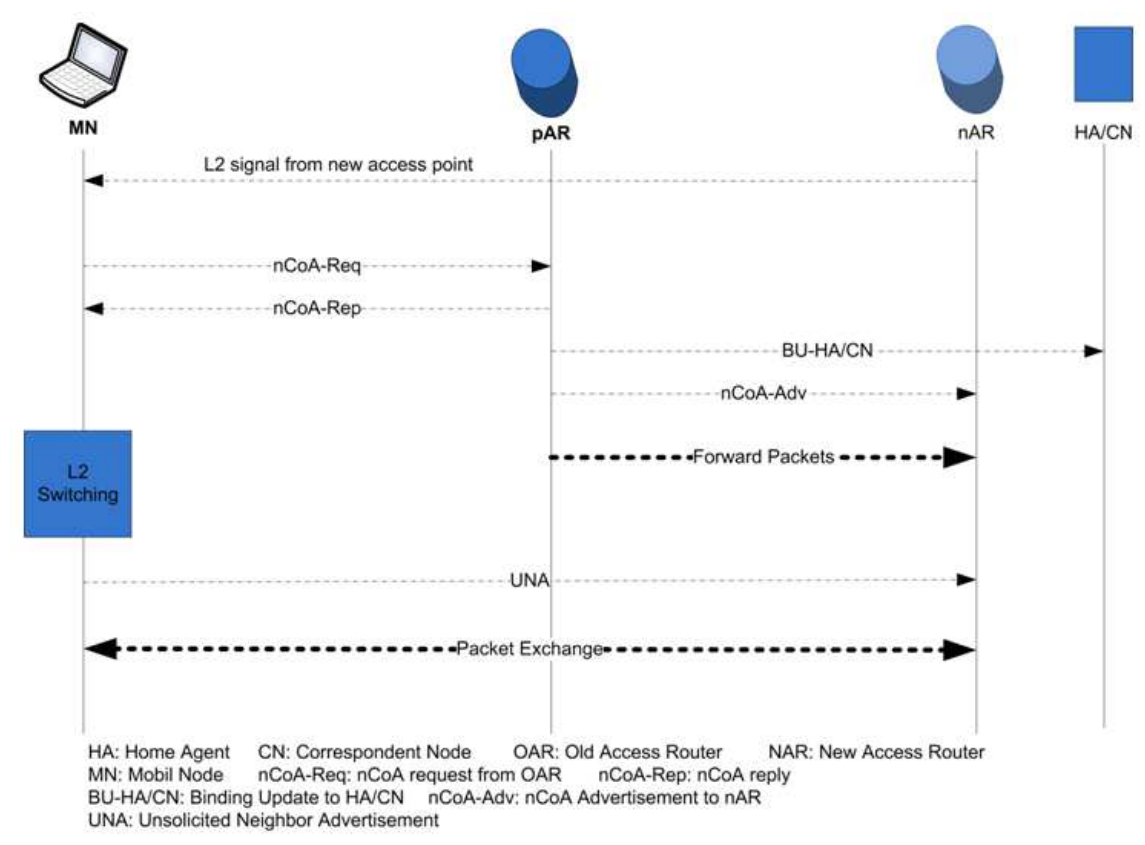

Fig. 3. Proposed handover method

\section{Method Operation}

The handover processes start as soon as the MN receives an L2 signal from one of the neighbouring networks. At that instant, the MN sends an nCoA-Req. message to the OAR including the L2 ID of the new network and a request option for a unique CoA. Upon receiving the nCoA-Req., by referring to the table, the OAR specifies the candidate network which the MN wants to handover to. Since the candidate AR is already specified, the OAR selects a unique CoA for the MN from the table depending on the new network prefix. It initiates an $\mathrm{BU}$ message for the unique address to the $\mathrm{HA} / \mathrm{CN}$. Also OAR sends an nCoA-Adv. message to neighbouring ARs, which have the same table of address in order to remove the used addresses. The OAR replies to the MN with a nCoA-Rep. including the new CoA of the candidate $\mathrm{AR}$ which corresponds to the existing L2 ID.

As soon as the MN receives an nCoA-Rep. message, it configures the address specified by the new CoA into its second interface without any delay. At the time that the MN moves towards the new network, the LGD trigger will be issued from the MIH to inform the IP layer of the link as a result of signal degradation in the old interface. Simultaneously a LGD trigger is also issued from the MIH to the IP layer in the OAR. This event will start the tunnel establishment between the OAR and the NAR. The MN activates the second interface and establishes a low layer connection to the NAR while disconnecting the old connection. This L2 handover is performed through a Link Switch (LS) command event which is received by the MIH layer at both the OAR and the MN. When the LU trigger is received right after the new connection is established, the MN is fully attached to the new network and sends an UNA message to the NAR to ensure the start of packet forwarding through the new connection as shown in Figure 3.

\section{Simulation and Results}

Figure 4 illustrates the network scenario. It consists of one WiMAX cell and one WLAN. The WLAN cell has partially overlapping with the WiMAX cell. UDP was used to transmit video and audio traffic from a $\mathrm{CN}$ to the MN. The data packet size was set to $4960 / 320$ bytes for video and audio respectively while the interpackets duration was fixed at $0.004 \mathrm{~s}$. The simulation time is set at 200s while the MN speeds is set between 1 $\mathrm{ms}$ and $25 \mathrm{~ms}$. The results given are the average of 10 simulations for every average speed simulated.

Figure 5 illustrates the handover latency for FMIPv6 compared to the proposed method. It is clear when using EA-DAD the handover operation can be significantly enhanced compared to FMIPv6. The average handover latencies for the proposed method were $0.39 \mathrm{~s}$ and $0.28 \mathrm{~s}$ for the video and audio respectively. While the FMIPv6 method was given the average handover latencies $1.14 \mathrm{~s}$ and $1.08 \mathrm{~s}$ for video and audio traffic. As a result, the EA-DAD mechanism is approximately reduced to overall handover latency of $0.77 \mathrm{~s}$. This is because the proposed method decreases the L3 handover latency which radically reduces the overall handover latency. 


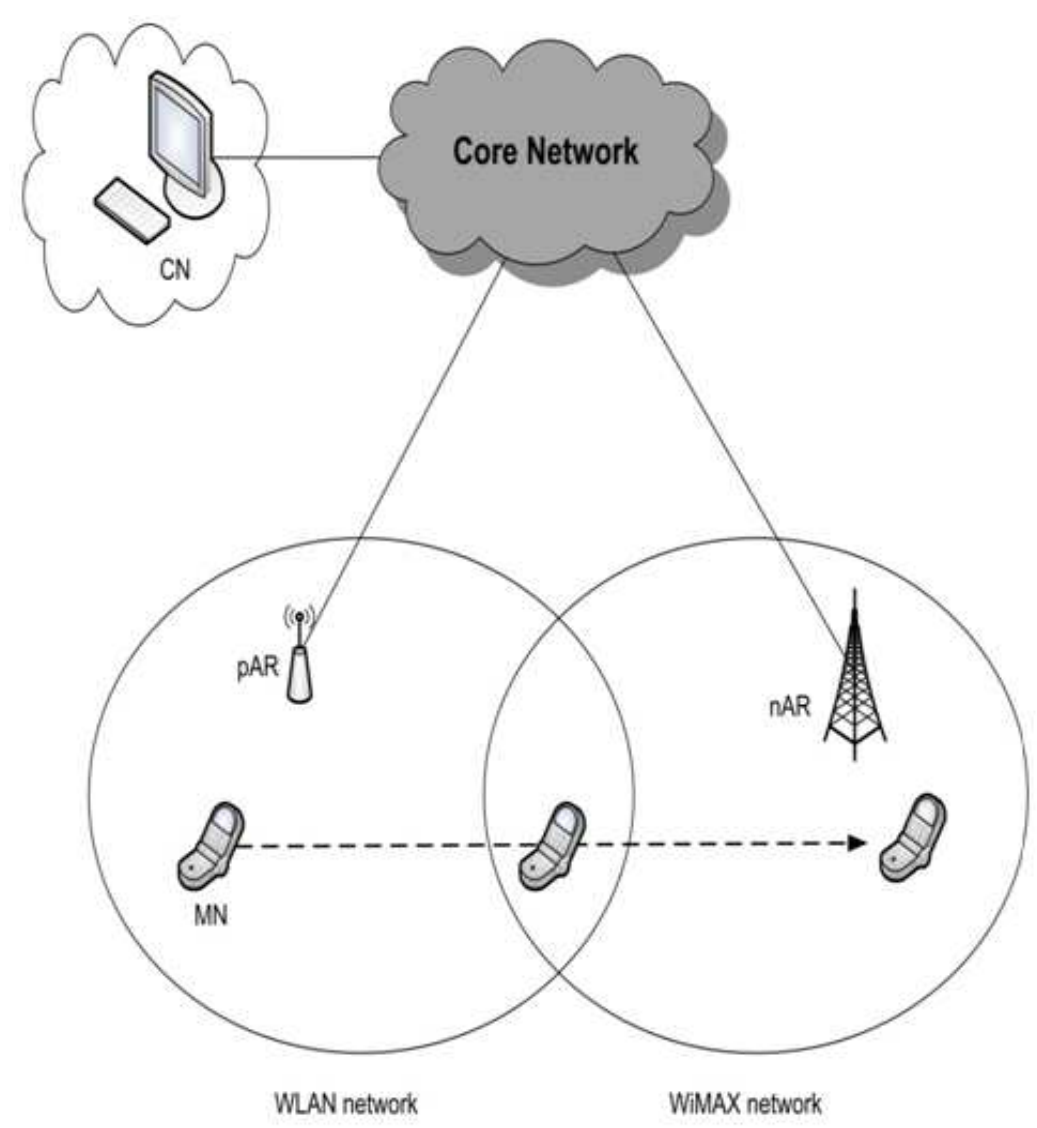

Fig. 4. The network scenario

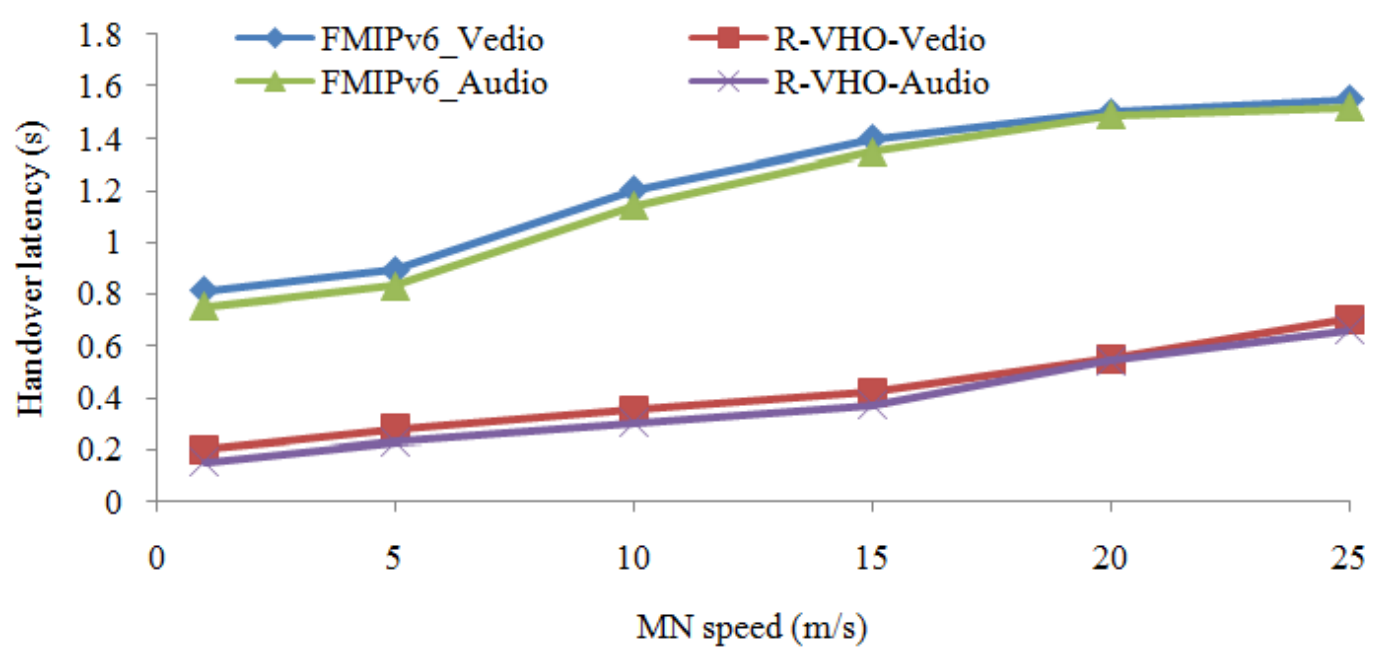

Fig. 5. Average handover latency Vs. node speeds

When the MN increases the speed, the handover latency in FMIPv6 and the EA-DAD method will also increase, although the maximum latency at a speed of $25 \mathrm{~ms}$ is $0.5 \mathrm{~s} / 0.38 \mathrm{~s}$ for video and audio respectively which are considered very little if compared to FMIPv6. This metric handover latency was used for comparison between the proposed method in (Vahid et al., 2008) and the FMIPv6. While the mentioned method gave an improved results by $40 \%$ compared to FMIPv6, our proposed method gave an improved results by $60 \%$ under the same circumstances. 


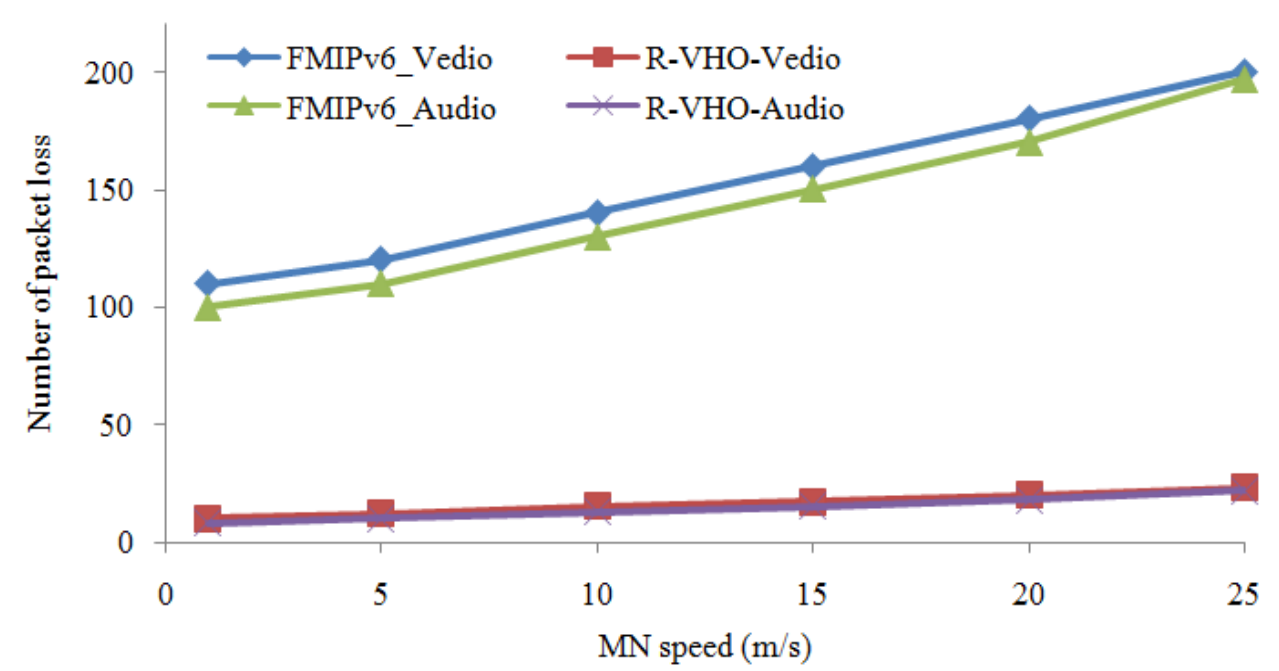

Fig. 6. Average packet loss Vs. node speeds

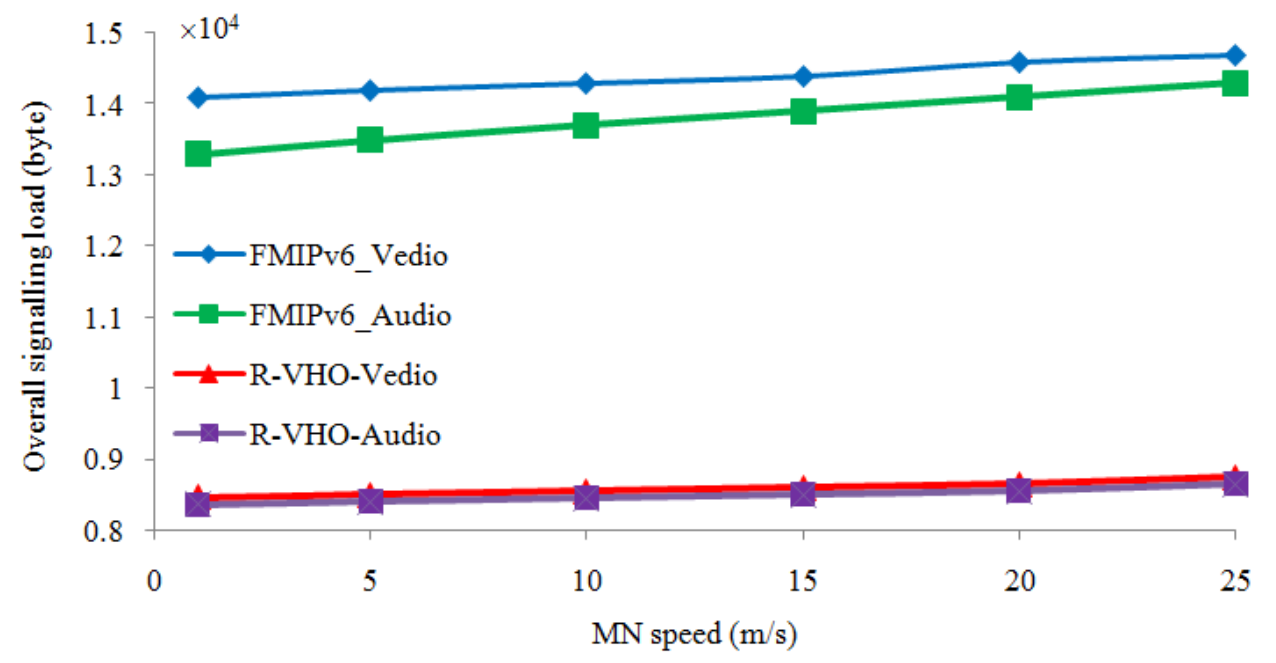

Fig. 7. Average overall messages signaling load

Figure 6 illustrates the packet loss for the FMIPv6 and R-VHO. Packet loss is defined as the amount of one or more data packets that are sent from the source $(\mathrm{CN})$ but did not receive from the destination (MN) in a limited time. As shown, packet loss with the FMIPv6 starts in 100 packets when the speed is $1 \mathrm{~ms}$ by audio traffic and overrides 200 at video traffic by highest speed $25 \mathrm{~ms}$, whereas the number for EA-DAD reaches 23 packet loss in a worst case. The R-VHO looses fewer packets compared to the FMIPv6 when the MN speed increases. The handover operation for FMIPv6 might not have enough time to complete the handover proceedings at the pAR link, when the speed of MN was high, therefore the data packets received through the $\mathrm{pAR}$ might be dropped. In addition, when the MN moves away from the pAR, the Received Signal Strength (RSS) and the Signal to Noise Ratio (SNR) decrease, thus the throughput of the connection is decreased causing more data packet losses. The method in (Vahid et al., 2008) gave an improved result in FMIPv6 by $75 \%$ to keep an average of 20 lost packets, our algorithm hardly reasoned 13 lost packets under the same circumstances and shows an improvement with an average of $83 \%$.

The overall messages signaling overhead represent the average signaling overhead (in bits) at the network layer and the above layers through the handover operations. Figure 7 illustrates the simulation results. The EA-DAD enhanced FMIPv6 by lessening up to $50 \%$ from the signaling overhead compared to the FMIPv6 in the vertical mode. This is mainly due to removing the RtSolPr, PrRtAdv and FBACK messages from the overall FMIPv6 handover signaling, which influence on decreasing the possibility of a reactive mode of handover. The EA-DAD assisted FMIPv6 in increasing 
the likelihood of a predictive mode of handover compared to the FMIPv6. Also notice when there are many MNs used in the test, the probability of increasing the signaling overhead will reduce due to the existence of the NT in the AR.

\section{Conclusion}

In this study, we proposed a new fast handover method called EA-DAD in which the exchange messages have been reduced by providing unique IP address to the $\mathrm{MN}$ from the OAR. At the same time, we proposed that binding updates to $\mathrm{HA} / \mathrm{CN}$ are performed by OAR. In our method we reduce the handover delay as well as preventing disruption of service when the MN moves at a fast speed during the handover excuation.

\section{Acknowledgement}

We thank our colleagues in Computer Science and Information Technology Department of Communication Technology and Network, UPM, Malaysia who provided insight and expertise that greatly assisted the research.

\section{Funding Information}

The authors have no support or funding to report.

\section{Author's Contributions}

All authors equally contributed in this work.

\section{Ethics}

This article is original and contains unpublished material. The corresponding author confirms that all of the other authors have read and approved the manuscript and no ethical issues involved.

\section{References}

Daley, G. and R. Nelson, 2003. Duplicate address detection optimization using IPv6 multicast listener discovery.

Dimopoulou, L., G. Leoleis and I.S. Venieris, 2005. Fast handover support in a WLAN environment: Challenges and perspectives. IEEE Netw., 19: 14-20. DOI: 10.1109/MNET.2005.1453394
Han, Y.H. and S.H. Hwang, 2006. Care-of address provisioning for efficient IPv6 mobility support. Comp. Commun., 29: 1422-1432. DOI: 10.1016/j.comcom.2005.09.002

IEEE, 2008. Draft standard for local and metropolitan area networks: Media independent handover services. Institute of Electrical and Electronics Engineers, USA.

Izuan, M. and A.Z. Zuriati, 2009. Performance analysis of random-based mobility models in MANET routing protocol. Eur. J. Scientific Res., 32: 444-454.

Johnson, D., C. Perkins and J. Arkko, 2004. RFC 3775mobility support in IPv6. Network working group.

Koodli, R., 2005. Fast handovers for mobile IPv6. Nokia Research Center.

Moore, N., 2006. Optimistic Duplicate Address Detection (DAD) for IPv6. RFC 4429.

Thomson, S., T. Narten and T. Jinmei, 2007. IPv6 Stateless Address Autoconfiguration. RFC 4862.

Tseng, C.C., Y.C. Wong, L.H. Yen and K.C. Hsu, 2006. Proactive DAD: A fast address-acquisition strategy for mobile IPv6 networks. IEEE Internet Comput., 10: 50-55. DOI: 10.1109/MIC.2006.131

Vahid, S., B.M. Ali, S. Khatun and D. Wong, 2008. Layer-2 protocol adaptation method to improve fast handoff for mobile IPv6 vertical handoffs, Proceedings of the 11th International Conference on Computer and Information Technology, Dec. 24-27, IEEE Xplore press, Khulna, pp: 492-497. DOI: $10.1109 /$ ICCITECHN.2008.4803008

Vivaldi, I., B.M. Ali, V. Prakash and A. Sali, 2003. Routing scheme for macro mobility handover in hierarchical mobile IPv6 network. Proceedings of the 4th National Conference on Telecommunication Technology, Jan. 14-15, IEEE Xplore press, pp: 88-92. DOI: $10.1109 /$ NCTT.2003.1188309 